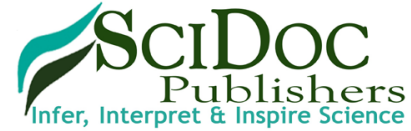

\title{
Use of HDFx, a Novel Immunomodulator, to Stop the Germs from Winning in Hospitals and on The Battlefields : The Dangers of Antibiotic Resistance \\ Editorial
}

\author{
Altura $\mathrm{BM}^{1-5^{*}}$, Altura $\mathrm{BT}^{1,3-5}$ \\ ${ }^{1}$ Department of Physiology \& Pharmacology, The State University of New York Downstate Medical Center, Brooklyn, New York, USA. \\ ${ }^{2}$ Department of Medicine, The State University of New York Downstate Medical Center, Brooklyn, New York, USA. \\ ${ }^{3}$ The Center for Cardiovascular and Muscle Research, The State University of New York Downstate Medical Center, Brooklyn, New York, USA. \\ ${ }^{4}$ The School of Graduate Studies in Molecular and Cellular Science, The State University of New York Downstate Medical Center, Brooklyn, New \\ York; \\ ${ }^{5}$ Bio-Defense Systems, Inc, Rockville Centre, New York, USA.
}

Getting admitted to a civilian or battlefield hospital these days often poses considerable risks and dangers. The ever-growing number of emerging diseases worldwide makes treatment of patients difficult and sometimes impossible. Many antibiotics are no longer effective against the simplest infections, which result in further hospitalizations with increased costs to the patients and governments worldwide. Microorganisms of prime concern include methicillin-resistant Staphylococcus aureus, Clostridium difficile, multidrug and extensive drug-resistant Mycobacterium tuberculosis, Neisseria gonorrhoeae, and carbapenem- resistant Enterobacteriaceae, as well as bacteria that produce extensive spectram beta-lactamases, such as E. coli. An unusual decline in the discovery of new and effective antibiotics, these days, is only making matters worse.

Despite government interventions, using diverse plans and some funding, the germs appear to be winning [1]. It is sad, to say, that governments, worldwide, are often concealing many dangerous outbreaks in numerous hospitals, including military service facilities. Deaths due to infections that could not be treated properly are often listed as due to respiratory or heart failure on the death-certificates. It is often stated that within three to four decades, these "superbugs" will kill more people, worldwide, than all deaths due to cancer and heart disease combined [1]. The World Health Organization has indicated that there has been a seven-fold increase in drug-resistant infections over the past eight years just among hospitalized children in the USA [1]. How can we reverse these deadly outcomes?

\section{HDFx: Discovery of a New Host-defense Immunomodulator}

For the past 30 years, our laboratories have been working on a new approach to develop host-defense factors that stimulate various arms of the innate and adaptive immune systems. To this end , we have discovered HDFx, a heretofore unknown host-defense factor in all mammals so far investigated (i.e., mice, rats, guineapigs, rabbits, dogs, and sub-human primates) [2-5]. We, thus, assume it is present in human subjects as well. About 135 years ago, Elie Metchnikoff, the father of immunology, hypothesized that the body, under stressful circumstances, would manufacture/ release molecules that could stimulate different arms of the innate immune system and serve to protect the host against major insults and diseases [6]. Metchnikoff's early studies pointed to the importance of macrophages and phagocytic leukocytes to natural (innate) resistance against pathogenic bacteria and viruses. During these past 30-40 years, a considerable number of studies on animals and humans, have demonstrated a strong relationship between the functional (physiological) state of the microcirculation, macrophages-leukocytes, natural killer (NK) cells, the reticuloendothelial system, and "pit" cells in the liver to host-defense and resistance to pathogens, trauma, circulatory shock, and sepsis [7-14].

Numerous experiments from our laboratories have clearly shown that HDFx is protective (to varying degrees) against a variety of systemic bodily insults ranging from hemorrhage, trauma, combined injuries, endotoxins, a variety of lethal bacteria (e.g., E. coli, S. enteriditis, C. welchii, among others), fungi (e.g., Aspergillis fumagatus), centripetal forces, and septic shock [2-5, 15-19]. An important attribute of HDFx is its unique ability to protect against "cytokine storms" in animals that are septic [19]; 'cytokine storms' are clearly known to be a major cause of lethality in hospitalized patients infected with numerous microorganisms (i.e., bacteria and fungi) who become resistant to antibiotic treatment [14]. It should be noted, here, that multiple virus infections, such as dengue, hanta viruses, diverse hemorrhagic fever viruses, and influenza

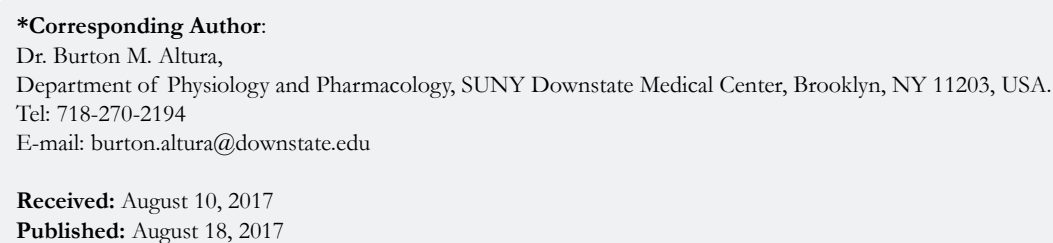

Copyright: Altura $\mathbf{B M}^{\circ}$ 2017. This is an open-access article distributed under the terms of the Creative Commons Attribution License, which permits unrestricted use, distribu tion and reproduction in any medium, provided the original author and source are credited. 
viruses can also trigger massive cell destruction, resulting in extensive "cytokine storms" which play pivotal roles in promoting lethality of patients in and out of hospitals, and on the battlefields [14]. To our knowledge, no other host-defense factor can stem the dissemination in the body of cytokines in sepsis, as can HDFx, at least experimentally in animals. Septic shock caused by severe bacterial/fungal infections accounts for about $10 \%$ of all human deaths in the U.S.A. each year, and is major cause of battlefield deaths and farm animal deaths each year.

\section{HDFx and "Super-Superbugs"}

Gran-negative "superbugs" seem to be the major culprits in many hospitalized patients and wounded military personnel on battlefields. Gram-negative bacteria are more difficult to kill than gram-positive bacteria because they are protected by "double membranes". So, in order to kill the gram-negative bacteria, most of the therapeutic approaches have been to design antibiotics to penetrate these membrane barriers. In our opinion, another likely approach would be to engulf the bacteria and digest them within "supercharged" macrophages, Kupffer cells, phagocytic leukocytes, "pit cells" and NK cells. HDFx appears, at least experimentally, to induce a "supercharged effect" in these various types of phagocytic cells in animals we have investigated to date. But for this kind of effect to take place, in a most expeditious fashion, we believe the microcirculation in the various regional vascular beds (i.e., liver, spleen, and lungs) must perforce produce optimal (physiologic) blood flows and distribution of capillary, nutritive flows. Fortunately, HDFx also posseses unique vasoactive properties which act to do exactly the latter in the microcirculation [2-5]. To our knowledge, no other known hostdefense factor, other than HDFx, can accomplish this task under septic conditions.

\section{Conclusions}

The discovery of HDFx may provide a unique way to ameliorate and prevent hospital-borne infections and be pivotal in the prevention of deaths, amputations, and disfigurement of wounded soldiers on the battlefield. We believe the approaches outlined, herein, and elsewhere [2-5, 15-18] regarding our discovery, or derivatives of HDFx, could be an ideal drug to pretreat all hospitalized patients and, especially, those scheduled for major surgeries.

\section{Acknowledgements}

Some of the original studies and thoughts needed for the discovery of HDFx and reviewed above, were initiated while the authors were at New York University School of Medicine (Department of Anesthesiology) and The Albert Einstein College of Medicine of Yehiva University (Departments of Anesthesiology \& Physiology). Some of the original studies reviewed, above, were supported, in part, by unrestricted grants from several pharmaceutical companies (CIBA-GEIGY Pharmaceuticals,
Sandoz Pharmaceuticals, Bayer Pharmaceuticals, and The UpJohn co.) and anonymous donors. The authors are indebted to many colleagues, over the years, who helped make our studies possible: C. Thaw, E.W. Burton, J. Hanley, C. Parillo, A. Carella, and A. Gebrewold. The authors are also grateful to the late Professor Solomon G. Hershey for several discussions over many years.

\section{References}

[1]. McCaughey B (2017) How the Feds are letting the germs win. New York Post. April 19, p 25.

[2]. Altura BM, Gebrewold A, Carella A (2009) A novel biologic immunomodulator, $\mathrm{HDFx}$, protects against lethal hemorrhage, endotoxins and traumatic injury: potential relevance to emerging diseases. Int J ClinExp Med 2(3): 266-279.

[3]. Altura BM, Carella A, Gebrewold A (2011) HDFx: a novel biologic immunomodulator is therapeutically effective in hemorrhagic and intestinalischemic shock: importance of microcirculatory-immunological interactions and their potential implications for the warfighter and disaster victims. Int J ClinExp Med 4(4): 331-340.

[4]. Altura BM, Carella A, Gebrewold A (2012) HDFx: A recently discovered immunomodulator accelerates wound healing and is suggestive of unique regerative powers: potential implications for the warfighter and disaster victims. Int J ClinExp Med 5(4): 289-295.

[5]. Altura BM, Gebrewold A, Carella A (2016) HDFx: A recently discovered biologic and its potential use in prevention and treatment of hemorrhagic fever viruses and antibiotic-resistant superbugs. J hematol Thromboembolic Dis 4(4): 100252.

[6]. Metchnikoff E (1884) Study on the intracellular digestion of turbulent animals. Works from the Zoological Institute of Vienna5(2): 141-168.

[7]. Altura BM (1980) Recent progress in pathophysiology of shock: Reticuloendothelial and neuro-endocrine stimulation. J ClinAnesth 4: 745.

[8]. Altura BM (1980) Reticuloendothelial cells and host defense. Adv Microcirculation 9:252-294.

[9]. Altura BM, Saba TM, eds(1981) Pathophysiology of the Reticuloendothelial System. Raven Press, New York.

[10]. Ulevitch RJ, Mathison JC, Tobias PS (1983) The role of the macrophage in host response to bacterial endotoxins. In: The Pathophysiology of Combined Injury and Shock.

[11]. Angele MK, Chaudry IH (2005) Surgical trauma and immunosupression: Pathophysiology and potential immunomodulatory approaches. Langebecks Arch Surg 390(4): 334-341.

[12]. Majno G, Joris I (2004) Cells, Tissues and Diseases. (2ndEdn). Oxford University Press, New York.

[13]. Caligiuri MA (2008) Human natural kiler cells. Blood 112(3): 461-469.

[14]. Murphy CK, Weaver C (2016) Janeway's Immunology. (9thEdn). Garland Science, New York.

[15]. Altura BM, Gebrewold A, Carella A, Altura BT (2016) HDFx: Potential new treatment and prophylactic against nonalcoholic steatohepatitis (NASH) and subsequent heptaticellular carcinomas: Is hypomagnesemia a -complication of the disease? Alcoholism and Drug Depend4(5): 47-58.

[16]. Altura BM, Gebrewold A, Carella A, Altura BT (2016) HDFx: A novel immunomodulator for the amelioration of hypovolemic shock in the OR and on the battlefield. J Clin Med Therap 1(1): e002.

[17]. Altura BM, Gebrewold A, Carella A, Altura BT (2016) HDFx: A novel biologic immunomodulator may have the potential to prevent bacteria in space from becoming aggressively infectious and lethal. Clin Res and Trials 3(3): $1-3$.

[18]. Altura BM, Gebrewold A, Carella A, Altura BT (2016) HDFx: A novel immunomodulator and potential fighter against cytokine storms in inflammatory and septic conditions in dogs and farm animals. Int J Vet Health Sci \& Res 5(2e): 1-3.

[19]. Altura BM, Altura BT (2017) HDFx: A novel biologic immunomodulator for potential control and treatment of NK cell and macrophage dysfunction in drug-resistant tuberculosis.J HematolThrombo Dis. 